\title{
Latent Fingerprint Matching: Fusion of Rolled and Plain Fingerprints
}

\author{
Jianjiang Feng, Soweon Yoon, and Anil K. Jain \\ Department of Computer Science and Engineering \\ Michigan State University \\ $\{$ jfeng, yoonsowo, jain\}@cse.msu.edu
}

\begin{abstract}
Law enforcement agencies routinely collect both rolled and plain fingerprints of all the ten fingers of suspects. These two types of fingerprints complement each other, since rolled fingerprints are of larger size and contain more minutiae, and plain fingerprints are less affected by distortion and have clearer ridge structure. It is widely known in the law enforcement community that searching both rolled and plain fingerprints can improve the accuracy of latent matching, but, this does not appear to be a common practice in law enforcement. To our knowledge, only rank level fusion option is provided by the vendors. There has been no systematic study and comparison of different fusion techniques. In this paper, multiple fusion approaches at three different levels (rank, score and feature) are proposed to fuse rolled and plain fingerprints. Experimental results in searching 230 latents in the NIST SD27 against a database of 4,180 pairs of rolled and plain fingerprints show that most of the fusion approaches can improve the identification performance. The greatest improvement was obtained by boosted max fusion at the score level, which reaches a rank-1 identification rate of $83.0 \%$, compared to the rank-1 rate of $57.8 \%$ for plain and $70.4 \%$ for rolled prints.
\end{abstract}

Keywords: Latent fingerprint, rolled fingerprint, plain fingerprint, fusion, minutiae matching.

\section{Introduction}

Automated Fingerprint Identification Systems (AFIS) have played an important role in forensic, law enforcement and many civilian applications. Fingerprint images in AFIS can be broadly classified into three categories, namely, (i) rolled, (ii) plain/flat and (iii) latent. Figure 1] shows these three types of fingerprint images from the same finger. Rolled fingerprint images are obtained by rolling a finger from one side to the other ("nail-to-nail") in order to capture all the ridge details of a finger. Plain fingerprints are those in which the finger is pressed down on a flat surface but not rolled. Rolled and plain impressions are obtained either by scanning the inked impression on paper or by directly using livescan devices. In AFIS, rolled and plain fingerprints are generally acquired in an attended mode and are subject to a recapture if the image quality is poor. In

M. Tistarelli and M.S. Nixon (Eds.): ICB 2009, LNCS 5558, pp. 695-704, 2009.

(C) Springer-Verlag Berlin Heidelberg 2009 


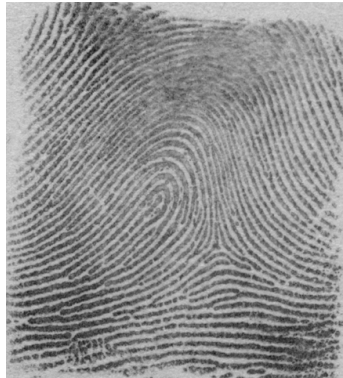

(a)

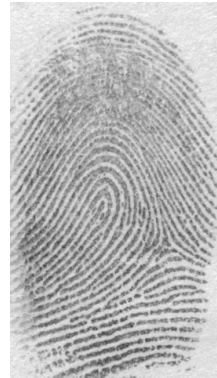

(b)

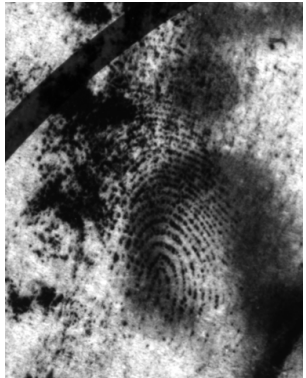

(c)

Fig. 1. Three types of fingerprint images. (a) Rolled fingerprint, (b) plain fingerprint and (c) latent fingerprint.

contrast, latent fingerprints are lifted from surfaces of objects that are inadvertently touched or handled by a person through a variety of means ranging from simply photographing the print to more complex dusting or chemical processing 1. With small area, unclear ridge structure, complex background and strong distortion, latent fingerprints generally have the worst image quality among the three types of fingerprints. However, it is the matching of a latent fingerprint against a database of rolled/plain fingerprints that is of utmost importance in forensics and law enforcement to apprehend suspects.

AFIS may work in automatic or semi-automatic mode depending on specific applications. In civil background check application, 10 finger impressions (rolled or plain) are submitted and the AFIS automatically returns the mated subject or reports no matches found. In suspect identification application, latents are submitted and the system generally returns a list of top candidates, which are then reviewed by latent experts.

Fingerprint image quality has a significant impact on system accuracy. Since the quality of latents is not controllable, it is important to ensure that enrolled fingerprints in the database have as clear ridge structure as possible. During the enrollment, the rolled and plain fingerprints of the ten fingers of a person are obtained through fourteen impressions. These impressions can be captured by using traditional tenprint cards or livescan devices. Traditional tenprint cards (see Fig. (2) contain the rolled impressions of the ten fingers as well as four slap impressions: the left slap (four fingers of the left hand), the right slap (four fingers of the right hand) and the thumb slaps (left and right thumbs). A segmentation algorithm is used to automatically segment the slaps into individual plain fingerprints. There are two reasons for including plain fingerprints in tenprint cards: (i) while rolled fingerprints contain larger size and larger number of minutiae, plain fingerprints are less distorted and often have clearer ridges; and (ii) out-of-sequence rolled fingerprints can be easily detected by matching rolled images to slap images [2]. With an aim to avoid the time-consuming and error prone rolling process, a touchless 3D fingerprint sensor was proposed in [3], which reconstructs 3D fingerprint using stereo vision techniques and then 


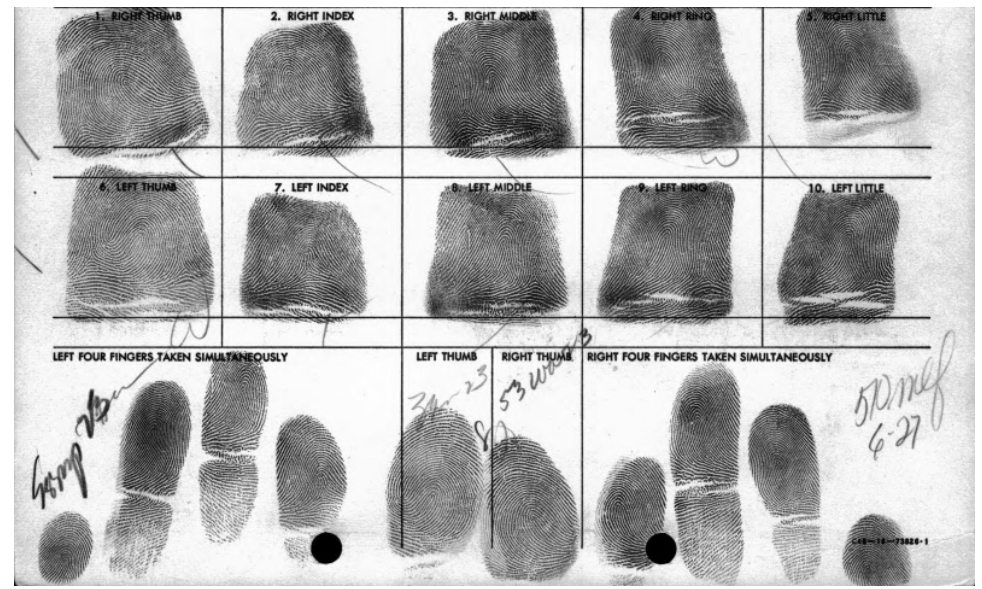

Fig. 2. A tenprint card from NIST SD29 consisting of fourteen fingerprint impressions

creates $2 \mathrm{D}$ rolled-equivalent fingerprints. While such a scanner is very desirable, the image quality provided by the current prototype of the scanner still needs significant improvement [4].

There have been some studies that compare matching accuracy using plain and rolled fingerprints. In the plain-to-rolled fingerprint matching experiments conducted by NIST [5], the identification rate of 10-finger plain-to-rolled and rolled-to-rolled is $97.5 \%$ and $97.9 \%$, respectively. The Federal Bureau of Investigation (FBI) [6] has conducted an experiment to evaluate the accuracy of matching latents against plain and rolled fingerprints. The hit rate of searching 250 latents in NIST SD27 against plain and rolled fingerprints in the FBI's Integrated Automated Fingerprint Identification System (IAFIS) is $38.8 \%$ and $54.4 \%$, respectively. Fusion at rank level leads to a hit rate of $61.2 \%$. Given this relatively low performance of latent fingerprint matching [78], there is a strong need for the improvement in the fusion of plain and rolled fingerprints for latent fingerprint matching.

Fusion of plain and rolled fingerprints is a type of multibiometric technique [9]. A multibiometric system can combine evidences from different sources, such as multiple fingers [10], multiple sensors [11, multiple samples [12] and multiple representations [13]. Fusing plain and rolled fingerprints can be viewed as fusing multiple samples of the finger. It can also be viewed as combining the impressions of the same finger captured by different sensors or sensing procedures. The evidences provided by different sources can be combined at different levels, such as score level [1013, feature level [12] and image level [1214]. Although it is generally believed that early stage fusion (image level) should provide better performance, fusion at the score level has received much more attention than fusion at the other levels. There are relatively few studies on fusion at the feature and image level in the literature 1214. In addition, no systematic work has been done to compare different fusion approaches at different levels for 
fingerprint identification, especially in the case of fusing plain and rolled fingerprints for latent matching.

In this study, multiple fusion approaches at each of the three different levels (rank, score and feature) are proposed to fuse rolled and plain fingerprints. These fusion approaches are evaluated according to the matching accuracy in searching latent fingerprints against rolled and plain fingerprints. Experimental results indicate that most of the fusion methods can improve the matching accuracy; the greatest improvement was obtained by boosted max fusion at the score level.

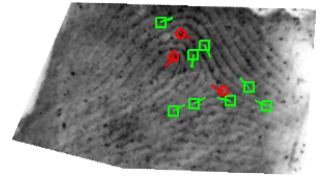

(a)

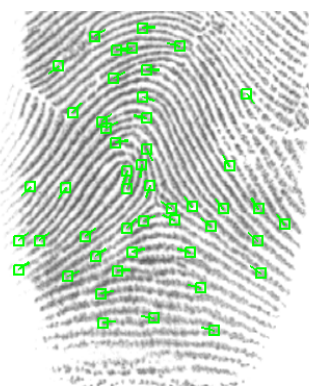

(b)

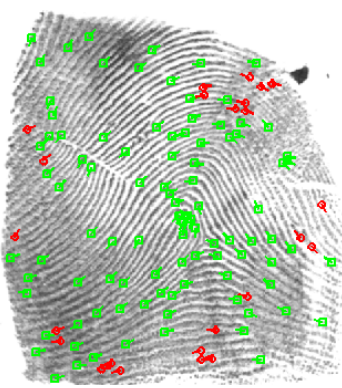

(c)

Fig. 3. (a) A latent fingerprint, (b) mated plain fingerprint, and (c) mated rolled fingerprint. Reliable minutiae are marked by green squares and unreliable ones are marked by red circles.

\section{Fusion Experiments}

\subsection{Database and Matcher}

Experiments were conducted on the new NIST SD27 database, which consists of 230 latents and 202 tenprint cards. The link between latents and the mated fingerprints was provided. The old NIST SD27 was not used, since it does not contain plain fingerprints. To increase the background database size, all 216 tenprint cards in set A of NIST SD29 were also used. Totally, the background database consists of 4,180 pairs of plain and rolled prints.

The features in the latents were manually marked, which is a common practice in the law enforcement community. Three types of features are used: minutiae, ridge orientation map and quality map. A minutia has four attributes: $x, y$, direction and quality. The minutia quality has two levels: reliable and unreliable. Ridge orientation map and quality map are defined on non-overlapping blocks of $16 \times 16$ pixels. The quality of a block has three levels: background, bad quality and good quality. The features of the plain and rolled fingerprints were automatically detected using the feature extraction algorithm in 8. Figure 3 shows a latent and the mated plain and rolled fingerprints (with minutiae marked).

The minutiae matching algorithm in [8] was used to match the 230 latents against the database containing 4,180 pairs of plain and rolled fingerprints. The 
Cumulative Match Characteristic (CMC) curves of of latent-to-plain and latentto-rolled matching are shown in Fig. 4. These two CMC curves are also included in all the subsequent figures as a baseline to evaluate fusion performance. A CMC curve plots the rank- $k$ identification rate against $k$, for $k=1,2, \cdots, 20$. The rank- $k$ identification rate indicates the proportion of times the mated fingerprint occurs in the top $k$ matches. The rank-1 identification rates of latent-to-plain and latent-to-rolled are $57.8 \%$ and $70.4 \%$, respectively. As expected, the accuracy of seaching latents against rolled images is much higher than against plain images. This phenomenon was also observed in the experiments conducted by FBI 6], where the rank-1 rates of latent-to-plain and latent-to-rolled matching are $38.8 \%$ and $54.4 \%$, respectively.

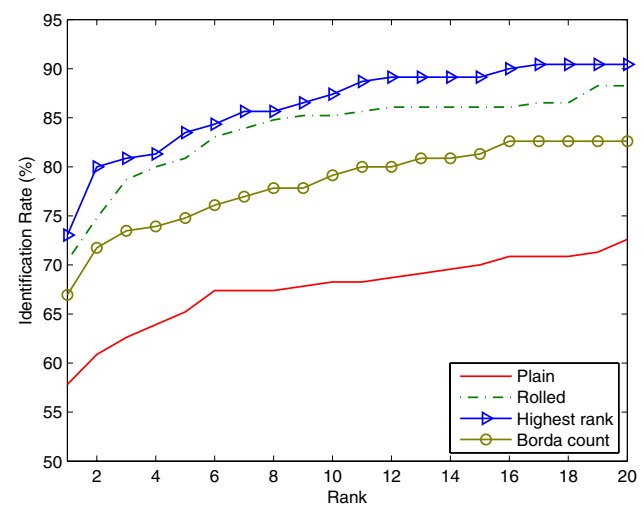

Fig. 4. The CMC curves of two rank level fusion methods: highest rank and Borda count

\subsection{Rank Level}

Two rank level fusion methods are adopted: highest rank and Borda count [15]. In the highest rank method, fingers are sorted with respect to the higher rank of plain and rolled fingerprints. If two fingers have the same higher rank, they are sorted by the lower rank of plain and rolled fingerprints. If both the higher and the lower ranks are the same, the two fingers are randomly sorted. The Borda count method uses the sum of the ranks of plain and rolled fingerprints to sort fingers. If the sums are the same, the two fingerprints are sorted by the higher rank of plain and rolled fingerprints. If both the higher and the lower ranks are the same, the two fingers are randomly sorted. The CMC curves of these two rank level fusion methods are given in Fig. 4. It can be observed that highest rank method is much better than Borda count. The accuracy of Borda count is even lower than that of latent-to-rolled matching. This is not surprising, since if either one of plain and rolled fingerprints has poor quality, the fused rank according to Borda count will be very low. In our experiments, we found that weighted Borda count also does not provide better performance. 


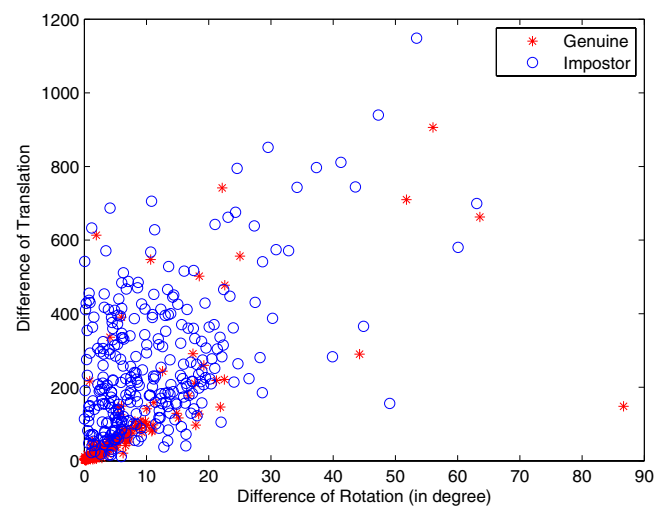

Fig. 5. The scatter plot of the rotation and translation differences of genuine matches and impostor matches

\section{$2.3 \quad$ Score Level}

Five score level fusion rules are tested: min, max, sum, product and boosted max. The purpose of the boosted max is to boost the score of genuine matches. Generally, for genuine matches, the spatial transformations among latent, plain and rolled fingerprints are consistent. But for impostor matches, the spatial transformations are generally not consistent. Let $T_{L P}, T_{L R}$ and $T_{P R}$ denote the rigid transformation matrices from latent to plain, latent to rolled and plain to rolled, respectively. These transformations are computed by our minutiae matcher. If the rigid transformation represented by $T_{L P R}=T_{L P} \times T_{P R}$ is similar to the rigid transformation represented by $T_{L R}$, the transformations among latent, plain and rolled fingerprints are viewed as consistent; otherwise they are viewed as inconsistent. Two rigid transformations, $T_{L P R}$ and $T_{L R}$, are said to be similar if the difference between the two rotations is less than $\pi / 8$ and the Euclidean distance between the two translations is less than 50 pixels. Figure 5 shows the scatter plot of the rotation and translation differences between $T_{L P R}$ and $T_{L R}$ for genuine matches and impostor matches. In the scatter plot, the genuine matches form a cluster near the origin, while the impostor matches scatter in the entire plane. The fused score according to boosted max rule is computed as:

$$
s_{F}=\left\{\begin{array}{ll}
\max \left(s_{P}, s_{R}\right)+w \cdot \min \left(s_{P}, s_{R}\right) & \text { if consistent } \\
\max \left(s_{P}, s_{R}\right) & \text { if inconsistent }
\end{array},\right.
$$

where $s_{P}$ and $s_{R}$ denote the scores of latent-to-plain and latent-to-rolled, respectively, and $w$ is a weight assigned to the minimum score, which has been set as 0.3 empirically. The CMC curves of these five score level fusion methods are given in Fig. 6. It can be observed that (i) all score level fusion rules provide improved performance except for the min rule, and (ii) boosted max gives the largest improvement, improving the rate-1 identification rate from $70.4 \%$ (rolled) and $57.8 \%$ (plain) to $83.0 \%$. The good performance of boosted max can be explained by the scatter plot in Fig. 5 . 


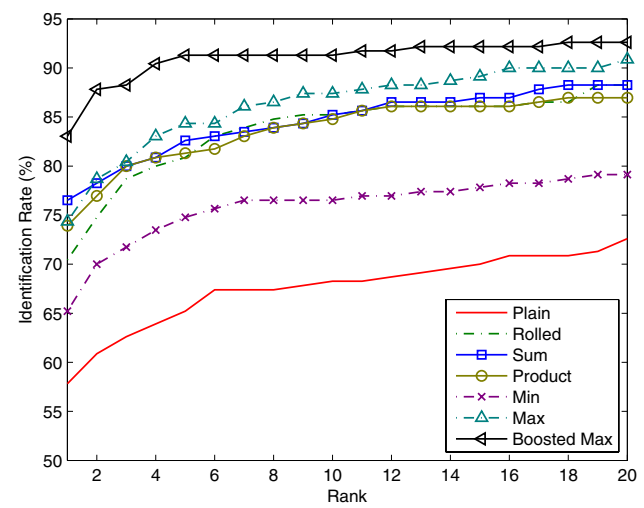

Fig. 6. The CMC curves of five score level fusion methods: sum, product, min, max and boosted max

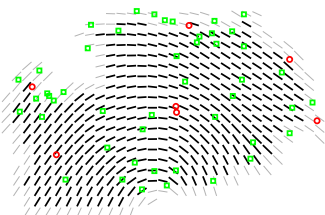

(a)

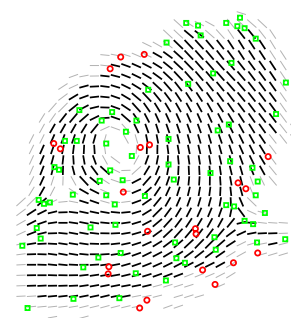

(b)

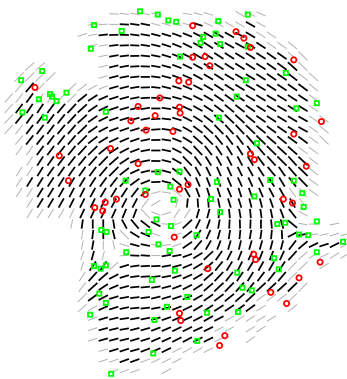

(c)

Fig. 7. Features of (a) the plain fingerprint, (b) the rolled fingerprint and (c) the composite fingerprint. Reliable minutiae are marked by green squares and unreliable ones are marked by red circles. Orientations in good quality regions are marked by dark lines and orientations in bad quality regions are marked by bright lines.

\subsection{Feature Level}

The plain and rolled fingerprints of the same finger are matched using our matcher. The output of the matcher includes a matching score, a set of matched minutiae, and the spatial transformation between the two images. The features of the plain fingerprint are mapped into the coordinate system of the rolled fingerprint. Three types of regions are identified: common region, plain-only region and rolled-only region. In the common region and the rolled-only region, the ridge orientation and quality of the rolled fingerprint are adopted. In the plain-only region, the ridge orientation and quality of the plain fingerprint are adopted.

Now we consider merging two sets of minutiae, minutiae from the plain fingerprint and minutiae from the rolled fingerprint, into one composite minutiae 


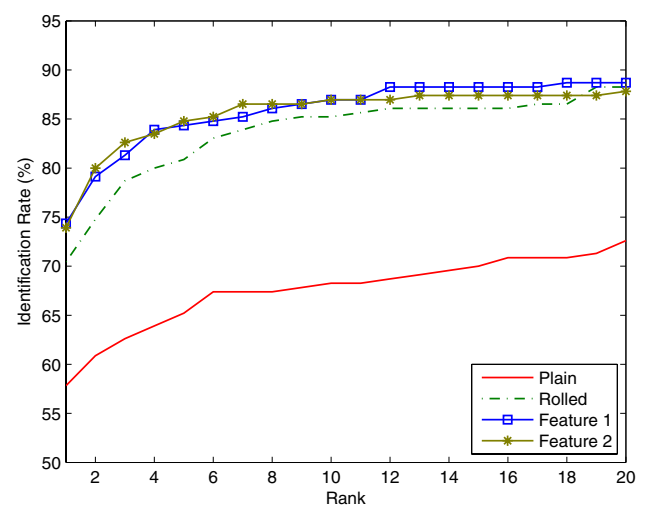

Fig. 8. The CMC curves of two different feature level fusion methods
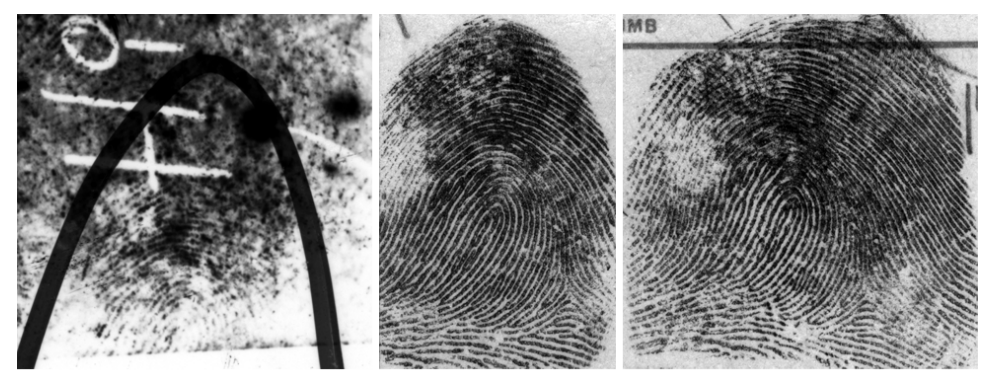

\begin{tabular}{|c|c|c|c|c|c|}
\hline & Plain & Rolled & $\begin{array}{c}\text { Highest } \\
\text { Rank }\end{array}$ & $\begin{array}{c}\text { Boosted } \\
\text { Max } \\
\end{array}$ & $\begin{array}{c}\text { Feature } \\
\text { Level }\end{array}$ \\
\hline Score & 0.39 & 0.30 & - & 0.46 & 0.42 \\
\hline Rank & 16 & 119 & 31 & 1 & 3 \\
\hline
\end{tabular}

Fig. 9. Fusion performance for a latent fingerprint and the mated plain and rolled fingerprints. Boosted max fusion gives the best result.

set. Minutiae that do not lie in the common region of the two fingerprints are directly used in the composite minutiae set. The minutiae in the common region may be either matched or unmatched. For matched minutiae, the minutiae in the rolled fingerprints are used and set as reliable if either of the two matched minutiae is reliable. The unmatched minutiae in the common region are treated in two different ways: (i) use all the unmatched minutiae, or (ii) if an unmatched minutia is reliable, set its quality as unreliable and include it in the composite minutiae set; if an unmatched minutia is unreliable, do not include it in the composite minutiae set. Figure 7 shows the features of a plain fingerprint, the mated rolled fingerprint and the composite fingerprint. The CMC curves of these two feature level fusion methods are given in Fig. 8. It can be observed that (i) both the methods provide improved performance and (ii) the first minutiae fusion method is slightly better than the second one. 
An example is given in Fig. 9 to compare the different fusion methods. When searching for the latent in Fig. 9 in the databases, the mated plain fingerprint gets a matching score of 0.39 and a rank of 16 , and the mated rolled fingerprint gets a matching score of 0.30 and a rank of 119. The higher rank of the plain image is because in the region that corresponds to the latent, the plain image has clearer ridge structures than the rolled image. Boosted max rule improves the mated finger to rank one and the feature level fusion improves it to rank three. The highest rank method gives a poor result due to the effect of ties.

\section{Conclusions and Future Work}

The problem of fusing rolled and plain fingerprints to improve the latent matching accuracy has been considered. Different fusion methods at three different levels (namely rank, score and feature) are studied. Experimental results in searching 230 latents against a database consisting of the plain and rolled fingerprints of 4,180 fingers indicate that boosted max provides the greatest improvement in identification accuracy.

The eventual goal of fusing rolled and plain fingerprints is to obtain full fingerprints of high quality. On one hand, new sensors or combination of sensors should be considered and on the other hand, robust fusion schemes should be explored based on existing data and sensors. Fingerprint mosaicking is a promising direction; however, simple image mosaicking using minutiae-based alignment is not likely to provide good performance. To develop a robust fingerprint mosaicking algorithm, the distortion between rolled and plain fingerprints must be accurately estimated, which is a challenging problem. We have only focused on fusing exemplar fingerprints in this work. Fusion of latent fingerprints is another opportunity for improving matching accuracy. For instance, a fingerprint mosaicking algorithm can be used to merge multiple latents left by the same finger into a single latent with higher image quality and larger image size.

\section{Acknowledgments}

We would like to acknowledge the support of Sagem Morpho and would like to thank Austin Hicklin, Noblis for providing us the new NIST SD27 database. This work was supported by ARO grant W911NF-06-1-0418 and NIJ grant 2007-RGCX-K183.

\section{References}

1. Lee, H.C., Gaensslen, R.E. (eds.): Advances in Fingerprint Technology. CRC Press, New York (2001)

2. Komarinski, P.: Automated Fingerprint Identification Systems (AFIS). Academic Press, London (2004) 
3. Parziale, G., Diaz-Santana, E.: The Surround Imager: a multi-camera touchless device to acquire 3D rolled-equivalent fingerprints. In: Zhang, D., Jain, A.K. (eds.) ICB 2006. LNCS, vol. 3832, pp. 244-250. Springer, Heidelberg (2006)

4. NIST: Evaluation of Fast Tenprint Capture Devices, http://fingerprint.nist. gov/FTcapture/index.html

5. Wood, S.S., Wilson, C.L.: Studies of plain-to-rolled fingerprint matching using the NIST algorithmic test bed (ATB). NISTIR 7112 (2004)

6. Swann, S.: Needs and applications of latents at FBI/CJIS, http://www.itl.nist. gov/iad/894.03/latent/workshop/proc/P6_Swann_LatentOverview_2.pdf

7. NIST: Summary of results from ELFT07 phase I testing (2007), http://fingerprint.nist.gov/latent/elft07/phase1_aggregate.pdf

8. Jain, A.K., Feng, J., Nagar, A., Nandakumar, K.: On matching latent fingerprints. In: Proc. CVPR Workshop on Biometrics, pp. 1-8 (2008)

9. Ross, A., Nandakumar, K., Jain, A.K.: Handbook of Multibiometrics. Springer, Heidelberg (2006)

10. Wilson, C., et al.: Fingerprint vendor technology evaluation 2003: Summary of results and analysis report. NISTIR 7123 (2004)

11. Marcialis, G.L., Roli, F.: Fingerprint verification by decision-level fusion of optical and capacitive sensors. In: Proc. ECCV Workshop on Biometric Authentication, pp. 307-317 (2004)

12. Jain, A.K., Ross, A.: Fingerprint mosaicking. In: Proc. International Conference on Acoustic Speech and Signal Processing (ICASSP), pp. 4064-4067 (2002)

13. Ross, A., Jain, A.K., Reisman, J.: A hybrid fingerprint matcher. Pattern Recognition 36(7), 1661-1673 (2003)

14. Hara, M., Toyama, H.: Print image synthesizing device and method, and print image synthesizing program. US Patent Application Publication No. 2006/002595A1 (2006)

15. Ho, T.K., Hull, J.J., Srihari, S.N.: Decision combination in multiple classifier systems. IEEE Trans. Pattern Analysis and Machine Intelligence 16(1), 66-75 (1994) 